\title{
Unsteady mixed convective MHD flow and mass transfer past an accelerated infinite vertical porous plate with suction
}

S.S. Das and M. Mitra ${ }^{1}$

Department of Physics, K B D A V College, Nirakarpur, Khurda-752 019, Orissa, India

${ }^{1}$ Ranpur College, Ranpur, Nayagarh-752 026, Orissa, India

drssd2@yahoo.com

\begin{abstract}
This paper analyzes the unsteady mixed convective mass transfer flow of a viscous incompressible electrically conducting fluid past an accelerated infinite vertical porous flat plate with suction in presence of a transverse magnetic field. The governing equations are solved both analytically and numerically using finite difference scheme. The flow phenomenon has been characterized with the help of flow parameters such as magnetic parameter $(M)$, suction parameter (a), Grashof number for heat and mass transfer $\left(G_{r}, G_{c}\right)$, Schmidt number $\left(S_{c}\right)$ and Prandtl number $\left(P_{r}\right)$. The effects of these parameters on the fluid velocity, temperature, concentration distribution, skin friction and heat flux have been analyzed and the results are presented graphically and discussed quantitatively. This type of problem is significantly relevant to geophysical, astrophysical and cosmical studies.
\end{abstract}

2000 Mathematics Subject Classification: 76W05

Keywords: Finite difference, MHD flow, mixed convection, mass transfer, accelerated plate, suction.

Introduction

The problem of mixed convective MHD flow with mass transfer has been given much importance because of its possible applications to nuclear research and in the study of stars and planets. These problems are of general interest in the field of geophysical and astrophysical studies. In view of these applications a series of investigations were made to study the flow past a vertical wall.

Convective flows under different physical situations have become a subject of interest of several researchers. Alam and Sattar (1999) analyzed the transient MHD heat and mass transfer flow in a rotating system in presence of thermal diffusion. Brinkman (1947) estimated the viscous force imparted by a flowing fluid in a dense swarm of particles. Chandran et al. (1998) studied the unsteady hydromagnetic free convection flow with heat flux and accelerated boundary motion. Choudhury and Das (2000) investigated the magnetohydrodynamic boundary layer flow of a non-Newtonian fluid past a flat plate. Das and his associates (2006) solved numerically the mass transfer effects on unsteady flow past an accelerated vertical porous plate

Fig A. Physical configuration and co-ordinate

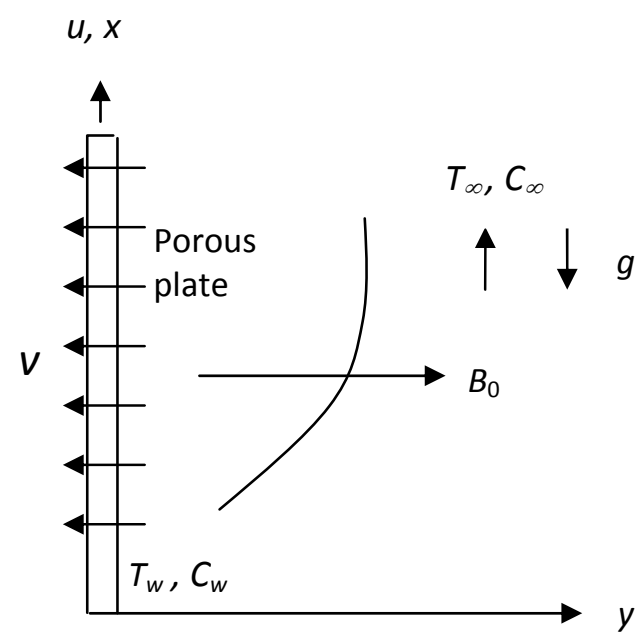

with suction. Dash and Das (1999) analyzed the effect of Hall current on MHD flow along an accelerated porous flat plate with mass transfer and internal heat generation. Hasimoto (1957) discussed the boundary layer growth on a flat plate with suction or injection. Makinde et al. (2003) have explained the unsteady free convection flow with suction on an accelerating porous plate.

Mansutti et al. (1993) have analyzed the steady flows of non-Newtonian fluids past a porous plate with suction or injection. Pathal and his co-workers (2005) discussed the unsteady mass, momentum and heat transfer in MHD free convection flow past a vertical plate suddenly set in motion. Raptis et al. (1987) have studied the unsteady free convective flow through a porous medium adjacent to a semiinfinite vertical plate using finite difference scheme. Sattar (1994) reported the free convection and mass transfer flow through a porous medium past an infinite vertical porous plate with time dependant temperature and concentration. Sharma and Pareek (2002) have discussed the steady free convection MHD flow past a vertical porous moving surface. Singh and Soundalgekar (1990) investigated the transient free convection effect in cold water past an infinite vertical porous plate. Soundalgekar (1974) analyzed the free convection effects on steady MHD flow past a vertical porous plate.

The study reported herein considers the unsteady mixed convective mass transfer flow of a viscous incompressible electrically conducting fluid past an accelerated infinite vertical porous flat plate with suction in presence of a transverse magnetic field. The governing equations are solved both analytically and numerically using finite difference scheme. The flow phenomenon has been characterized with the help of flow parameters and the effects of these parameters on the velocity field, temperature field, skin friction and heat flux have been analyzed and the results are presented graphically and discussed quantitatively with the help of graphs and tables. This type of problem has some relevance in geophysical, astrophysical and cosmical studies. Mathematical formulation

Consider the unsteady mixed convective mass transfer flow of a viscous incompressible electrically conducting fluid past an accelerating vertical infinite porous 
flat plate in presence of a transverse magnetic field $B_{0}$. The physical configuration and co-ordinate system of the problem is shown in Fig. A. Let the $x$-axis be directed upward along the plate and the $y$-axis normal to the plate. Let $u$ and $v$ be the velocity components along $x$ - and $y$ axes respectively. We assume that the plate is accelerating with a velocity $\mathrm{u}=\mathrm{Ut}$ in its own plane at time $t \geq 0$.Then the magnetohydrodynamic unsteady mixed convective boundary layer equations under usual Boussinesq's approximation are:

$$
\begin{aligned}
& \frac{\partial v}{\partial y}=0 \\
& \frac{\partial u}{\partial t}+v \frac{\partial u}{\partial y}=v \frac{\partial^{2} u}{\partial y^{2}}+g \beta\left(T-T_{\infty}\right)+g \beta *\left(C-C_{\infty}\right)-\frac{\sigma B_{0}^{2}}{\rho} u \\
& \frac{\partial T}{\partial t}+v \frac{\partial T}{\partial y}=k \frac{\partial^{2} T}{\partial y^{2}} \\
& \frac{\partial C}{\partial \mathrm{t}}+v \frac{\partial C}{\partial \mathrm{y}}=\mathrm{D} \frac{\partial^{2} \mathrm{C}}{\partial \mathrm{y}^{2}}
\end{aligned}
$$

where, $v$ is the kinematic viscosity, $k$ is the thermal diffusivity, $\beta$ is the volumetric coefficient of expansion for heat transfer, $\beta^{*}$ is the volumetric coefficient of expansion for mass transfer, $\rho$ is the density, $\sigma$ is the electrical conductivity of the fluid, $g$ is the acceleration due to gravity, $T$ is the temperature, $T_{\infty}$ is the temperature of the fluid far away from the plate, $C$ is the concentration, $\mathrm{C}_{\infty}$ is the concentration of the fluid far away from the plate and $D$ is the molecular diffusivity.

The necessary boundary conditions are,

$$
\begin{aligned}
& \mathrm{u}=U t, T=T_{w}, C=\mathrm{C}_{w} \text { at } y=0, \\
& \mathrm{u} \rightarrow 0, T \rightarrow T_{\infty}, C \rightarrow C_{\infty} \text { as } y \rightarrow \infty \text { for } t \rightarrow \infty
\end{aligned}
$$

We introduce the similarity variables and dimensionless quantities

$$
\begin{aligned}
& \eta=\frac{y}{2 \sqrt{v t}}, u=U t f(\eta), \theta=\frac{T-T_{\infty}}{T_{w}-T_{\infty}}, C=\frac{C-C_{\infty}}{C_{w}-C_{\infty}}, \\
& M=\frac{\sigma B_{0}^{2}}{\rho} \frac{v}{v^{2}}, \\
& P_{r}=\frac{v}{k}, S_{c}=\frac{v}{D}, G_{r}=4 g \beta \frac{T_{w}-T_{\infty}}{U} \\
& G_{c}=4 g \beta * \frac{C_{w}-C_{\infty}}{U},
\end{aligned}
$$

where $P_{r}=$ Prandtl number,

$\mathrm{G}_{\mathrm{r}}=\mathrm{Grashof}$ number for heat transfer,

$\mathrm{G}_{\mathrm{c}}=$ Grashof number for mass transfer,

$M=$ magnetic parameter,
$\mathrm{S}_{\mathrm{c}}=$ Schmidt number and the primes denote the derivative with respect to $\eta$.

Following Hasimoto (1957), Das et al. (2006) and Singh and Soundalgekar (1990), we choose

$v=-a\left(\frac{v}{t}\right)^{\frac{1}{2}}$,

where $a>0$ is the suction parameter.

Using equations (6) and (7), equations (2) - (4) become

$f^{\prime \prime}+2(\eta+a) f^{\prime}-4\left(1+a^{2} M\right) f=-G_{r} \theta-G_{c} C$,

$\theta^{\prime \prime}+2(\eta+a) P_{r} \theta^{\prime}=0$

$C^{\prime \prime}+2(\eta+a) S_{c} C^{\prime}=0$.

The boundary conditions now take the form

$f(0)=1, C(0)=1, f(\infty)=0, \theta(\infty)=0, C(\infty)=0$.

Skin friction

The skin friction at the wall is given by

$\tau=\rho v\left(\frac{\partial u}{\partial y}\right)=-\rho U \frac{\sqrt{v t}}{2} f^{\prime}(0)$.

In non-dimensional form, we get

$\tau^{\prime}=\frac{2 \tau}{\rho U \sqrt{v t}}=-f^{\prime}(0)$.

Heat transfer

The non-dimensional local heat flux in terms of Nusselt number $\left(\mathrm{N}_{\mathrm{u}}\right)$ at the plate is given by

$N_{u}=\frac{2 q_{w} \sqrt{v t}}{k\left(T_{w}-T_{\infty}\right)}=-\theta^{\prime}(0)$,

where $q_{w}$ is the heat flux per unit area.

Method of solution

Solving equations (9) and (10) exactly by error function subject to boundary conditions (11), we get

$$
\begin{gathered}
\theta=\frac{\operatorname{erfc}\left((\eta+a) \sqrt{P_{r}}\right)}{\operatorname{erfc}\left(a \sqrt{P_{r}}\right)}, \\
C=\frac{\operatorname{erfc}\left((\eta+a) \sqrt{S_{c}}\right)}{\operatorname{erfc}\left(a \sqrt{S_{c}}\right)} .
\end{gathered}
$$

Equation (8) is solved employing finite difference scheme. In order to solve equation (8) by this method, we set up the following difference approximations:

$$
f^{\prime}=\frac{f_{i+1}-f_{i-1}}{2 h}, f^{\prime \prime}=\frac{f_{i+1}-2 f_{i}+f_{i-1}}{h^{2}} \text {. }
$$

Introducing these difference approximations (17) in equation (8), we obtain

$$
A_{0} f_{i+1}+A_{1} f_{i}+A_{2} f_{i-1}=B_{1}+B_{2} \text {, }
$$




$$
\begin{gathered}
\text { where } A_{0}=\frac{1+h\left(\eta_{i}+a\right)}{h^{2}}, \\
A_{1}=-\frac{2+4 h^{2}\left(1+a^{2} M\right)}{h^{2}}, \\
A_{2}=\frac{1-h\left(\eta_{i}+a\right)}{h^{2}}, \\
B_{1}=-G_{r} \theta_{i}, \quad B_{2}=-G_{c} C_{i} . \\
\eta_{i}=i h, h=\frac{L}{N+1}, 0 \leq \eta_{i} \leq L .
\end{gathered}
$$

We take $L=2.0$ and $N=200$, since it lies well outside the boundary layer.

Results and discussions

The problem of unsteady mixed convective mass transfer MHD flow past an accelerated infinite vertical porous flat plate with suction has been formulated, analyzed and solved both analytically and numerically using error function and finite difference scheme. The effects of the flow parameters such as magnetic parameter (M), suction parameter (a), Grashof number for heat and mass transfer $\left(\mathrm{G}_{\mathrm{r},} \mathrm{G}_{\mathrm{c}}\right)$, Schmidt number $\left(\mathrm{S}_{\mathrm{c}}\right)$ and Prandtl number $\left(P_{r}\right)$ on the velocity, temperature and concentration profiles of the flow field are presented with the help of velocity profiles (Fig.1-4), temperature profiles (Fig.5, 6) and concentration profiles (Fig.7, 8). The nondimensional skin friction at the wall and the local heat flux in terms of Nusselt number are also discussed and entered in Table 1.

The velocity of the flow field varies to an appreciable extent with the variation of the flow parameters. The flow parameters affecting the velocity of the flow field are magnetic parameter $(M)$, suction parameter (a), Grashof number for heat and mass transfer $\left(G_{r}, G_{c}\right)$, Schmidt number $\left(\mathrm{S}_{\mathrm{c}}\right)$ and Prandtl number $\left(\mathrm{P}_{\mathrm{r}}\right)$. The effects of these parameters on the velocity of the flow field are analyzed with the help of Fig.1-4. The velocity profiles are in good agreement with the results of Das et al. (2006).

Fig. 1 shows the effect of suction parameter (a) on the velocity profiles of the flow field. The suction parameter is found to retard the velocity of the flow field at all points. The reduction in velocity at any point of the flow field is faster as the suction parameter becomes larger. One interesting inference of this finding is greater suction leads to a faster reduction in the velocity of the flow field.

Table 1. Variation of heat flux $\left(N_{u}\right)$ and skin friction

\begin{tabular}{|c|c|c|}
$\left(\tau^{\prime}\right)$ with'a' for $P_{r}=0.71, M=1$, Gr $=1$ and $G_{c}=1$ \\
\hline$a$ & $\mathrm{~N}_{u}$ & $\tau^{\prime}$ \\
\hline 0.1 & 1.04299514 & 0.198389241 \\
\hline 1.0 & 2.00275564 & 2.84287185 \\
\hline 2.0 & 3.23732738 & 6.200361849 \\
\hline
\end{tabular}

Fig.2 depicts the effect of magnetic parameter (M) on the velocity of the flow field. Keeping the other parameters of the flow field constant, the magnetic parameter is varied and its effect is studied. The magnetic parameter is found to retard the velocity at all points of the flow field. Higher the parameter, the sharper is the reduction in velocity of the flow field.

The effect of Grashof number for heat transfer $\left(G_{r}\right)$ on the velocity of the flow field is shown in Fig.3. Here, the velocity of the flow field is plotted against $\eta$ for four different values of the Grashof number keeping other parameters of the flow field constant. A study of the curves of the said figure shows that the Grashof number for heat transfer $\left(G_{r}\right)$ accelerates the velocity of the flow field at all points.

Fig. 4 depicts the effect of Grashof number for mass transfer $\left(G_{c}\right)$ on the velocity of the flow field. Comparing the curves of the said figure, it is observed that the Grashof number for mass transfer has an accelerating effect on the velocity of the flow field at all points.

The temperature of the flow field varies vastly with the variation of suction parameter (a) and the Prandtl number $\left(P_{r}\right)$. These variations in the temperature of the flow field are shown in Fig.5 and 6. The temperature profiles closely agree with the results of Das et al. (2006).

Fig. 5 presents the temperature profiles against $\eta$ for four different values of the suction parameter (a) keeping $P_{r}$ as constant. The suction parameter is found to decrease the temperature of the flow field at all points. It is further observed that cooling of the plate is faster as the suction parameter becomes larger. Thus, larger suction leads to faster cooling of the plate.

Fig. 6 shows the plot of temperature of the flow field against $\eta$ for different values of Prandtl number $\left(P_{r}\right)$ taking $a$ as constant. Comparing the curves of Fig.6, it is observed that the temperature of the flow field decreases in magnitude as $P_{r}$ increases. Thus, higher Prandtl number $\left(P_{r}\right)$ leads to faster cooling of the plate.

The concentration distribution of the flow field is found to change more or less with the variation of suction parameter (a) and the Schmidt number $\left(S_{c}\right)$. These variations are shown graphically in Fig.7 and 8 respectively. The concentration profiles agree with the results obtained in case of Das et al. (2006).

Fig.7 depicts the variation in the concentration distribution of the flow field against the non-dimensional distance $\eta$ for four different values of the suction parameter. The suction parameter is found to decrease the concentration boundary layer of the flow field at all points. Greater suction leads to a sharper reduction in the concentration boundary layer of the flow field.

The variation in the concentration boundary layer of the flow field due to different values of Schmidt number is shown in Fig.8. The effect of Schmidt number is to decrease the concentration distribution of the flow field. It is further found that the decrease in the concentration boundary layer of the flow field is more significant in presence of heavier diffusing species.
Research article

CIndian Society for Education and Environment (iSee)
"MHD flow"

http://www.indjst.org
Das \& Mitra Indian J.Sci.Technol. 
The heat flux in terms of Nusselt number $\left(\mathrm{N}_{\mathrm{u}}\right)$ and the non-dimensional skin friction $\left(\tau^{\prime}\right)$ are entered in Table 1 for different values of suction parameter (a). The suction parameter is found to enhance the heat flux and the skin friction at the wall. Conclusions

The above study brings out the following inferences of physical interest on the velocity, temperature and the concentration distribution of the flow field:

1. Greater suction (a) leads to a faster reduction in the velocity of the flow field.

2. The magnetic parameter $(M)$ retards the velocity of the flow field at all points.

3. The Grashof numbers for heat transfer $\left(G_{r}\right)$ and mass transfer $\left(G_{c}\right)$ have accelerating effect on the velocity of the flow field at all points.

4. Heavier diffusing species has a significant decrease in the concentration boundary layer of the flow field. The Schmidt number $\left(S_{c}\right)$ and the suction parameter (a) have a retarding effect on the concentration distribution of the flow field.

5. At any point in the flow field, the cooling of the plate is faster as the suction parameter (a) and Prandtl number $\left(P_{r}\right)$ become larger. Thus greater suction/ Prandtl number leads to faster cooling of the plate.

6. The effect of suction parameter is to enhance both the skin friction $\left(\tau^{\prime}\right)$ and the heat flux $\left(\mathrm{N}_{\mathrm{u}}\right)$ at the wall.

\section{References}

1. Alam MM and Sattar MA (1999) Transient MHD heat and mass transfer flow with thermal diffusion in a rotating system. J. Energy, Heat and Mass Transfer. 21, 9-21.

2. Brinkman HC (1947) A calculation of viscous force extended by flowing fluid in a dense swarm of particles. Appl. Sci. Res. A (1), 27-34.

3. Chandran P, Sacheti NC and Singh AK (1998) Unsteady hydromagnetic free convection flow with heat flux and accelerated boundary motion. $J$. Phys.Soc. Japan. 67, 124-129.

4. Choudhury R and Das A (2000)

Magneto-hydrodynamic boundary layer flows of non-Newtonian fluid past a flat plate. Ind. J. Pure Appl. Math. 31(11), 1429-1441.

5. Das SS, Sahoo SK and Dash GC (2006) Numerical solution of mass
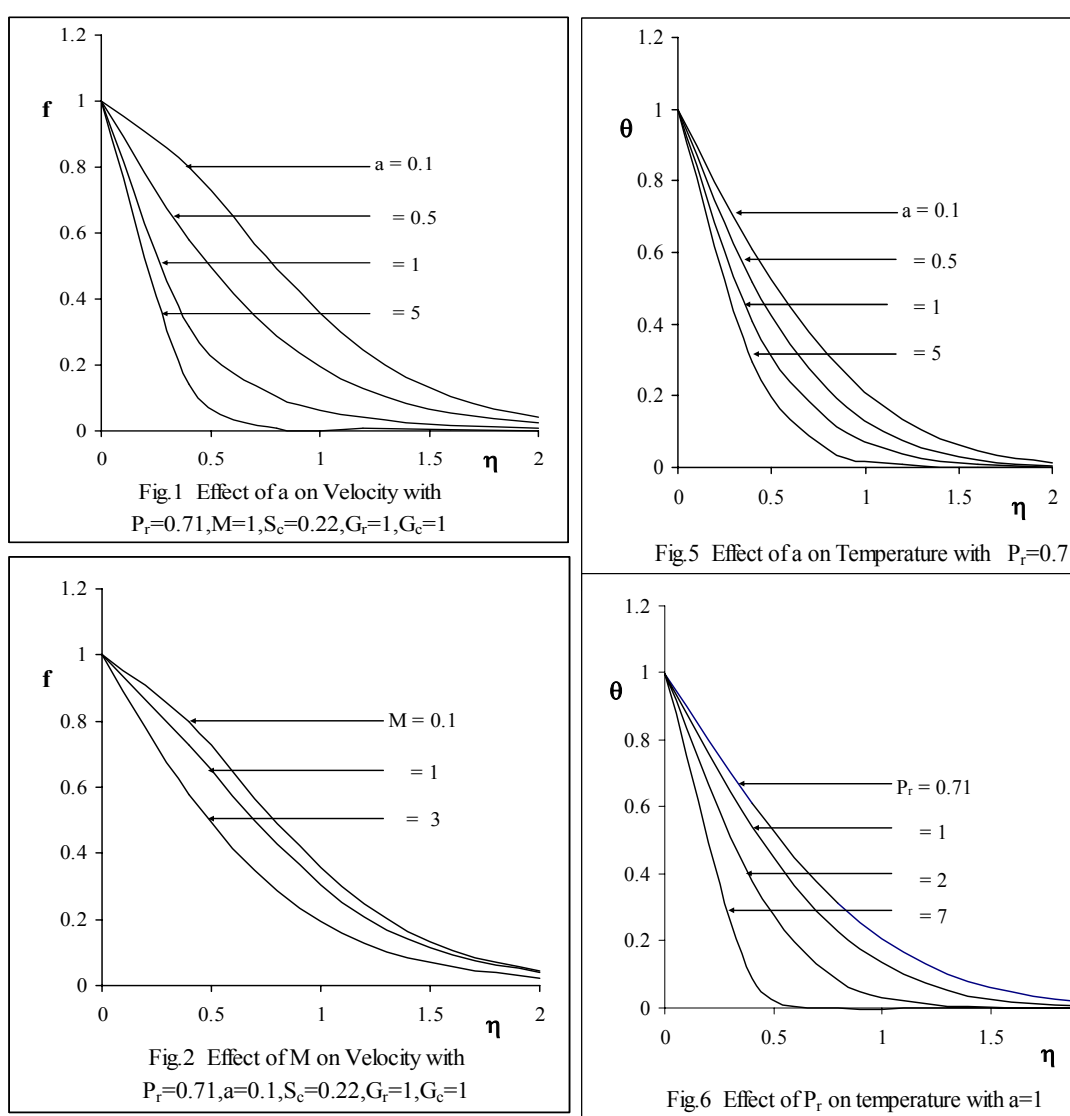

Fig.5 Effect of a on Temperature with $\mathrm{P}_{\mathrm{r}}=0.71$

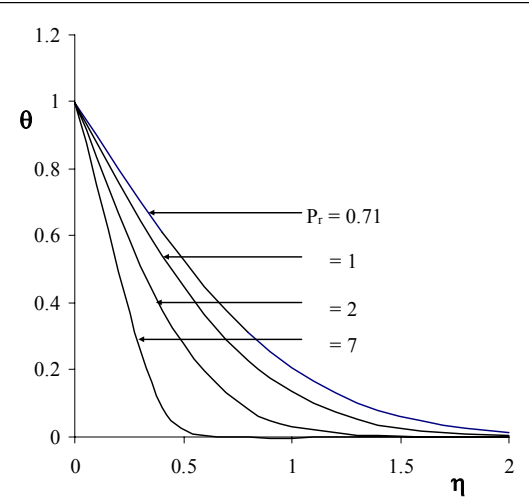

Fig.6 Effect of $\mathrm{P}_{\mathrm{r}}$ on temperature with $\mathrm{a}=1$

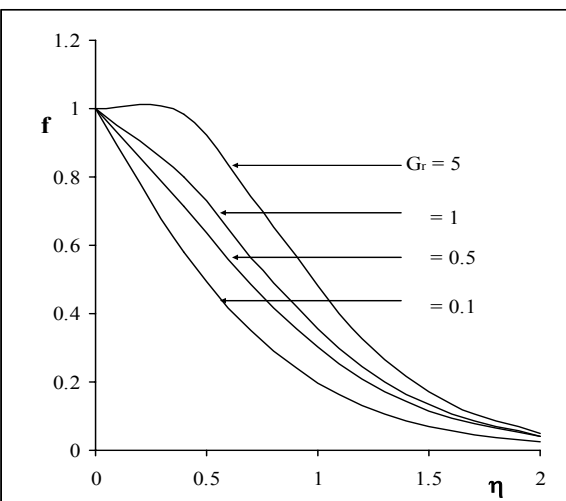

Fig. 3 Effect of $\mathrm{G}_{\mathrm{r}}$ on Velocity with $\mathrm{P}_{\mathrm{r}}=0.71, \mathrm{a}=0.1, \mathrm{~S}_{\mathrm{c}}=0.22, \mathrm{M}=1, \mathrm{G}_{\mathrm{c}}=1$
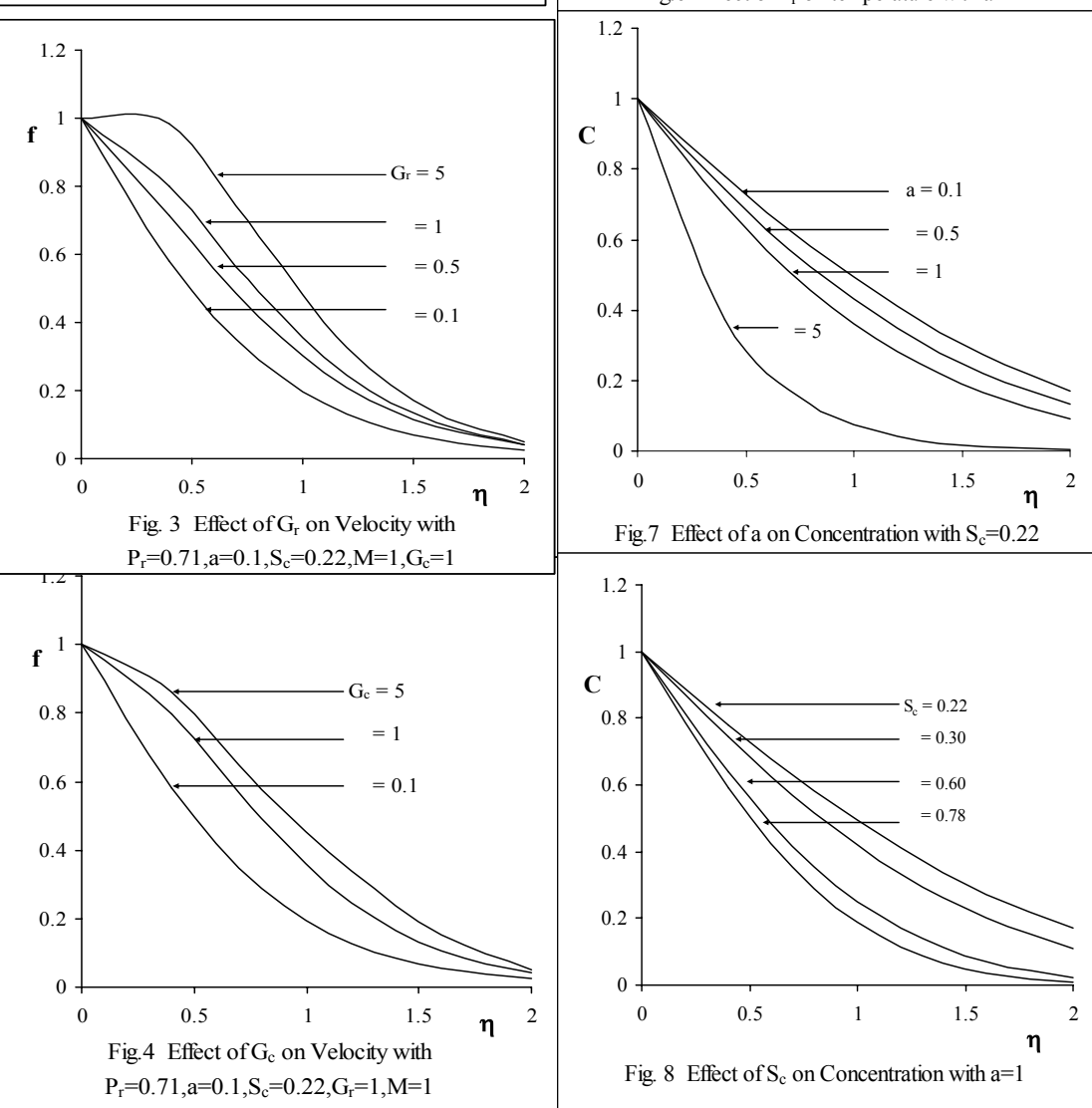

Fig. 7 Effect of a on Concentration with $\mathrm{S}_{\mathrm{c}}=0.22$

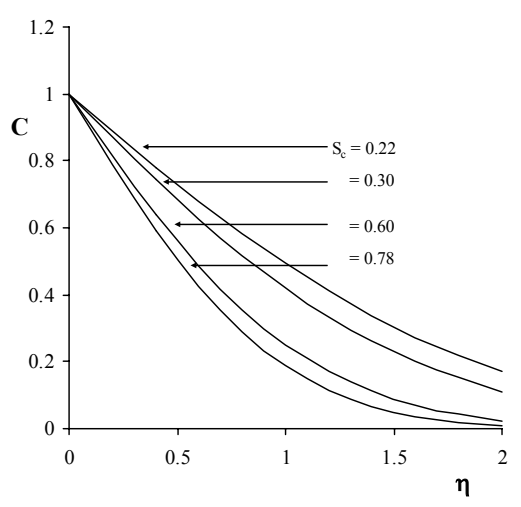

Fig. 8 Effect of $\mathrm{S}_{\mathrm{c}}$ on Concentration with $\mathrm{a}=1$ 
transfer effects on unsteady flow past an accelerated vertical porous plate with suction. Bull. Malays. Math. Sci. Soc. 29(1), 33-42.

6. Dash GC and Das SS (1999) Hall effects on MHD flow along an accelerated porous flat plate with mass transfer and internal heat generation. Math. Engng. Indust. 7(4), 389-404.

7. Hasimoto $H$ (1957) Boundary layer growth on a flat plate with suction or injection. J. Phys. Soc. Japan. 12, 68-72.

8. Makinde OD, Mango JM and Theuri DM (2003) Unsteady free convection flow with suction on an accelerating porous plate. AMSE J. Mod. Meas. Cont. B. 72(3), 39-46.

9. Mansutti D, Pontrelli G and Rajagopal KR (1993) Steady flows of non-Newtonian fluids past a porous plate with suction or injection. Int. J. Num. Methods Fluids. 17, 927-941.

10. Pathal G, Maheswari C and Tak SS (2005) Unsteady mass, momentum and heat transfer in MHD free convection flow past along a vertical plate suddenly set in motion. Bull. Pure Appl. Sci. E. 24(1), 173-183.

11. Raptis A, Singh AK and Rai KD (1987) Finite difference analysis of unsteady free convective flow through a porous medium adjacent to a semi-infinite vertical plate. Mech. Res. Comm. 14, 9-16.

12. Sattar MA (1994) Free convection and mass transfer flow through a porous medium past an infinite vertical porous plate with time dependant temperature and concentration. Ind. J. Pure Appl. Math. 23, 759-766.

13. Sharma PR and Pareek D (2002) Steady free convection MHD flow past a vertical porous moving surface. Ind.J.Theo.Phys. 50, 5-13.

14. Singh AK and Soundalgekar VM (1990) Transient free convection in cold water past an infinite vertical porous plate. Int. J. Energy Res. 14, 413-420.

15. Soundalgekar VM (1974) Free convection effects on steady MHD flow past a vertical porous plate. J. Fluid Mech. 66, 541-551. 